\title{
Fixation of os acromiale using polyester sutures: a novel surgical treatment
}

\author{
Dun-Ming Guo, Zu-Xi Li, Qing Wang, Huang-He Song \\ Department of Orthopedics, the First Affiliated Hospital of Nanjing Medical University, Nanjing 210029, China \\ Contributions: (I) Conception and design: All authors; (II) Administrative support: All authors; (III) Provision of study materials or patients: All \\ authors; (IV) Collection and assembly of data: All authors; (V) Data analysis and interpretation: All authors; (VI) Manuscript writing: All authors; (VII) \\ Final approval of manuscript: All authors. \\ Correspondence to: Huang-He Song. Professor, Department of Orthopedics, the First Affiliated Hospital of Nanjing Medical University, Nanjing \\ 210029, China. Email: songhuanghe@foxmail.com.
}

\begin{abstract}
Background: Patients with unstable os acromiale often complain of shoulder pain. Numerous surgical treatment options have been introduced with inconsistent clinical results. In this study, a novel surgical treatment using polyester sutures to fix unstable os acromiale was introduced, and clinical results were reported.

Methods: We retrospectively studied 10 shoulders that were diagnosed with os acromiale from January 2014 to January 2016. All 10 cases were of the meso-acromion type. Except for the first case in our series, cases of os acromiale were fixed using polyester sutures arthroscopically. The standardized scores and visual analog scale (VAS) were recorded preoperatively and at each follow-up. A computed tomography (CT) scan was ordered at the follow-up of 12 months.

Results: The average follow-up length was 28.7 months, ranging from 26 to 33 months. The average Constant score before surgery was $40.50 \pm 4.53$ points, which significantly improved to $75.60 \pm 5.17$ points after surgery. The average VAS score was reduced from $5.20 \pm 1.14$ points to $1.60 \pm 0.84$. At the follow-up of 12 months, a CT scan was ordered. All the patients showed a bony union of the os acromiale. On the CT scan, two small pits could be seen on the medial and lateral side of the acromion, which indicated the level of the os acromiale. The position of the os acromiale was good, and no evident sclerosis was found on the edges of the fragments.
\end{abstract}

Conclusions: Polyester sutures could provide reliable strength for the fixation of os acromiale without any irritation from hardware.

Keywords: Shoulder; os acromiale; arthroscopy

Submitted Mar 08, 2019. Accepted for publication Aug 08, 2019.

doi: $10.21037 /$ atm.2019.08.58

View this article at: http://dx.doi.org/10.21037/atm.2019.08.58

\section{Introduction}

Gruber first described os acromiale in a cadaver study in 1863 (1). The acromion develops from 4 separate ossification centers: the pre-acromion, meso-acromion, meta-acromion, and basi-acromion from anterior to posterior. The fusion of the 4 ossification centers occurs between the age of 15 and 25 years (2). Os acromiale is the failure of fusion between any of the 4 ossification centers after the age of 25 years.

The real incidence of this phenomenon was unknown because os acromiale usually shows no symptoms and is found incidentally on radiographic examinations (3). The reported incidence ranged from $1 \%$ to $30 \%$, and bilateral os acromiale incidence ranged from $33 \%$ to $62 \%$ (4-6).

Patients with unstable os acromiale often complain of shoulder pain, and the symptoms usually include difficulty sleeping on the affected side during the night and troubles 
Table 1 Diagnosis and treatments

\begin{tabular}{lll}
\hline Patient No. & Diagnosis & Treatments \\
\hline $1^{*}$ & Os acromiale, rotator cuff tear, frozen shoulder & Fixation, subacromial debridement, capsular release, rotator cuff repair \\
2 & Os acromiale & Fixation, subacromial debridement \\
3 & Os acromiale, rotator cuff tear & Fixation, subacromial debridement, rotator cuff repair \\
4 & Os acromiale, rotator cuff tear & Fixation, subacromial debridement, rotator cuff repair \\
5 & Os acromiale & Fixation, subacromial debridement \\
6 & Os acromiale, biceps tendon inflammation & Fixation, subacromial debridement, biceps tenotomy \\
7 & Os acromiale, frozen shoulder & Fixation, subacromial debridement, capsular release \\
8 & Os acromiale, frozen shoulder & Fixation, subacromial debridement, capsular release \\
9 & Os acromiale, rotator cuff tear & Fixation, subacromial debridement, rotator cuff repair \\
10 & Os acromiale & Fixation, subacromial debridement \\
\hline
\end{tabular}

*, for patient No. 1, rotator cuff repair was carried out arthroscopically while a small incision was made for fixation of os acromiale.

doing overhead activities, which are quite similar symptoms as those found in rotator cuff tears and subacromial impingement. Non-steroidal anti-inflammatory drugs (NSAIDs) are initially prescribed, along with physical therapy. If non-surgical treatments are not effective after 6 months, surgical treatments are considered (6). In the literature, numerous surgical treatment options have been introduced, including fragment excision, open reduction and internal fixation (ORIF) with $\mathrm{K}$-wires or screws, and arthroscopic subacromial decompression with acromioplasty (7-9). In this study, a novel surgical treatment using polyester sutures to fix unstable os acromiale is introduced, and clinical results are reported.

\section{Methods}

We retrospectively studied 10 shoulders that were diagnosed with os acromiale from January 2014 to January 2016. All of the patients received oral NSAIDs for at least 6 months without any relief from shoulder pain. There were two males and eight females, with an average age of 62 years ranging from 57 to 66 years at the time of surgery. The diagnosis and treatments for each patient are presented in Table 1. All 10 cases were of the meso-acromion type.

All patients were followed up for at least 24 months (average 28.7 months; range, 26 to 33 months). The clinical charts and surgery reports, including arthroscopic images and photos taken during the operations, preoperation, and follow-up radiographs, were all reviewed. The standardized scores and visual analog scale (VAS) were recorded preoperatively and at each follow-up. A computed tomography (CT) scan was ordered at the follow-up of 12 months.

\section{Surgical treatments}

The first patient of our case series was a 62-year-old female who complained of right shoulder pain after a trauma. On physical examination, there was tenderness over the acromioclavicular (AC) joint. The patient showed a limited range of motion, both actively and passively. They also returned positive results for Neer sign, Hawkins sign, and empty can test. Magnetic resonance imaging (MRI) revealed a partially-torn supraspinatus tendon and adhesive capsulitis, which agreed with the symptoms and physical examination findings. After 6 months of conservative treatment without significant relief, the patient underwent surgical treatment. A routine diagnostic arthroscopy was carried out, and a partial bursa-sided rotator cuff tear with capsulitis was confirmed. After a subacromial debridement, os acromiale was revealed, which was unexpected before the surgery. During the operation, we reviewed the magnetic resonance (MR) images and found the os acromiale on very proximal and lateral cuts (Figure 1) from the axial plane and oblique sagittal plane images, which were overlooked on preoperative estimation.

After arthroscopic repair of the rotator cuff and release of the capsule, we decided to make a small incision over the acromion to fix the os acromiale. After complete exposure of the acromion, we found that the bone quality was 

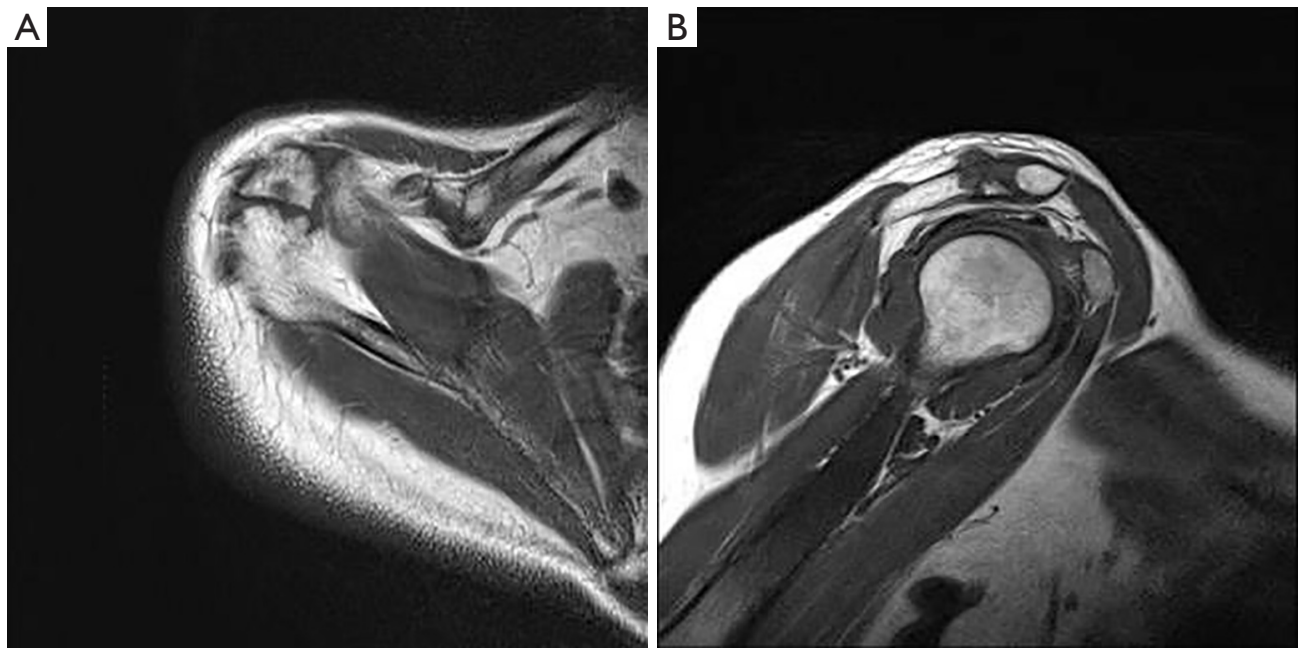

Figure 1 MR images. (A) Os acromiale on axial plane; (B) os acromiale on oblique sagittal plane.

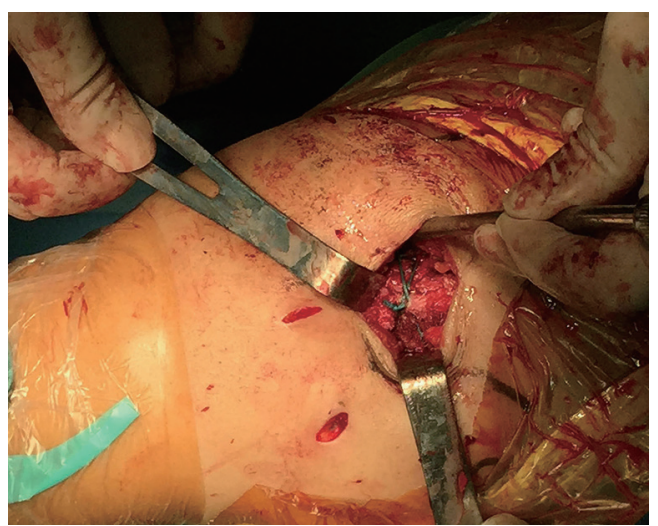

Figure 2 Open reduction of os acromiale and fixation with polyester sutures.

reduced for the 62-year-old woman. Screw fixation or metal cerclage with wires seemed difficult. Consequently, we used two \#5 Ethibond sutures (Ethicon, Somerville, NJ, USA) instead to reduce the risks of bone cuttings from the metal fixations. Two sutures were used for cerclage from anterior to posterior, and proper fixation was confirmed during the operation (Figure 2).

For the other nine shoulder cases, os acromiale was diagnosed before surgery and was confirmed during a routine diagnostic bursoscopy. Arthroscopic fixation of os acromiale using polyester sutures was applied, which has thus far not been reported in previous studies. The patient was put in a lateral decubitus position. With the arthroscope placed in the subacromial bursa through the posterior portal, the os acromiale could be identified. A radiofrequency device was used to remove the soft tissues underneath the acromion so that the os acromiale was wholly exposed. Following exposure of the undersurface of the acromion, a rasp was used to prepare the two fragments (Figure 3A). Sufficient punctate bleeding on both sides of the two fragments was important for the bony fusion of the os acromiale (Figure 3B). A spinal needle was put percutaneously from the posterior board of the acromion and underneath it (Figure 3C). A PDS II suture was shuttled through and grasped out of the lateral portal. The PDS II suture was used as a guide, and one end of a \#5 Ethibond suture was pulled in from the lateral portal and outside posteriorly. The step was repeated from the anterior board. Two \#5 Ethibond sutures were used (Figure 3D, Figure 4A). The Ethibond sutures were retrieved from a small incision of about $5 \mathrm{~mm}$ made above the acromion (Figure $4 B$ ), and knots were made using a knot pusher. The arthroscopic view demonstrated that the margin between the two fragments was gone (Figure 4C).

As listed in Table 1, there were three cases diagnosed as os acromiale alone without frozen shoulder, rotator cuff tears, SLAP lesion, or biceps tendon lesion. For the other seven cases, arthroscopic rotator cuff repair was carried out in four cases accompanied with rotator cuff tears, capsular release in three cases with frozen shoulder. In one case, inflammation of the biceps tendon was diagnosed. Considering the patient was a 64-year-old female, we cut the tendon from the 

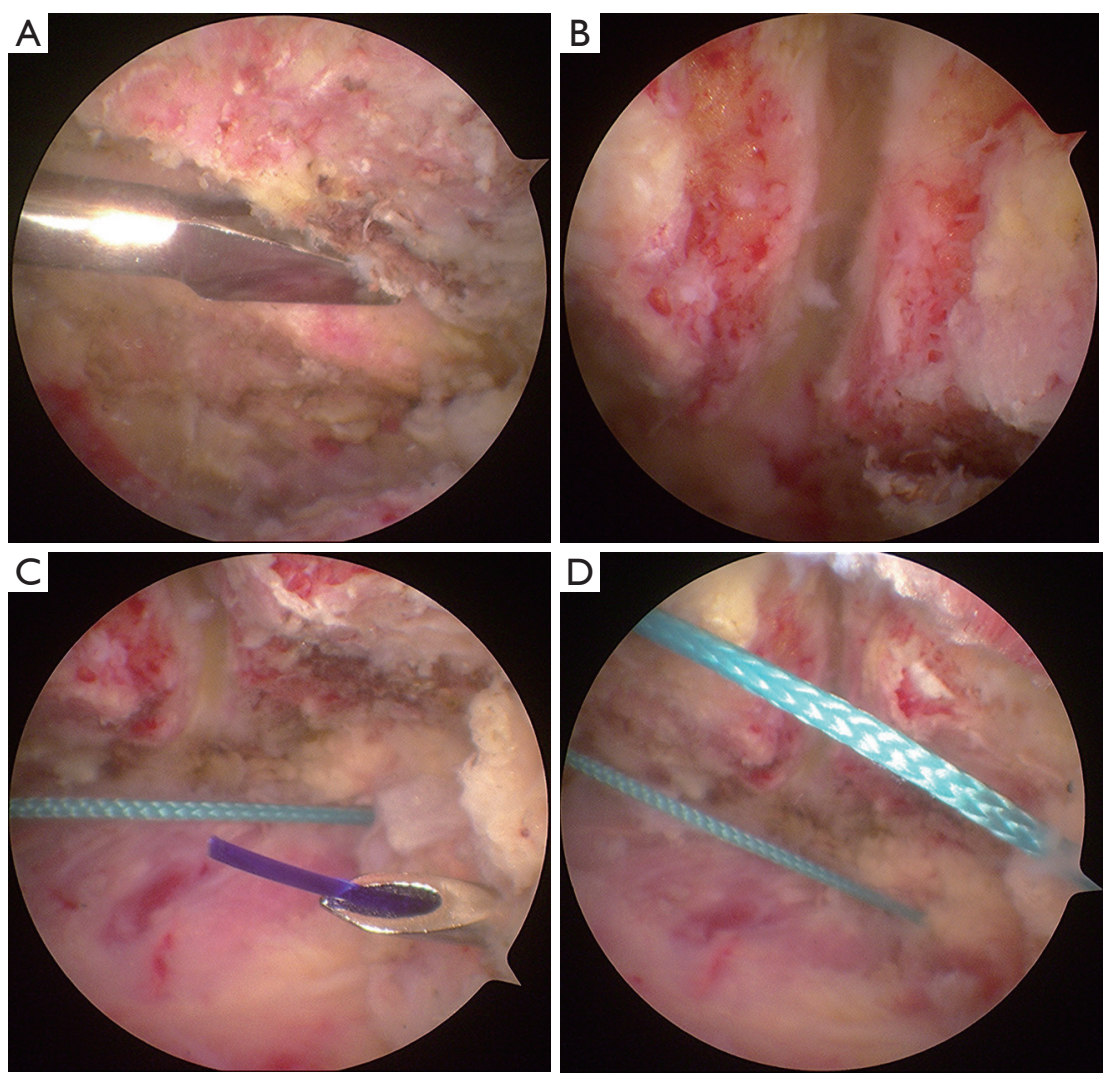

Figure 3 Arthroscopic fixation of os acromiale. (A) A rasp was used to prepare the two fragments; (B) evident punctate bleeding was seen on both edges; (C) A PDS II was shuttled through a spinal needle underneath the acromion; (D) arthroscopic view of the two Ethibond sutures underneath the acromion.
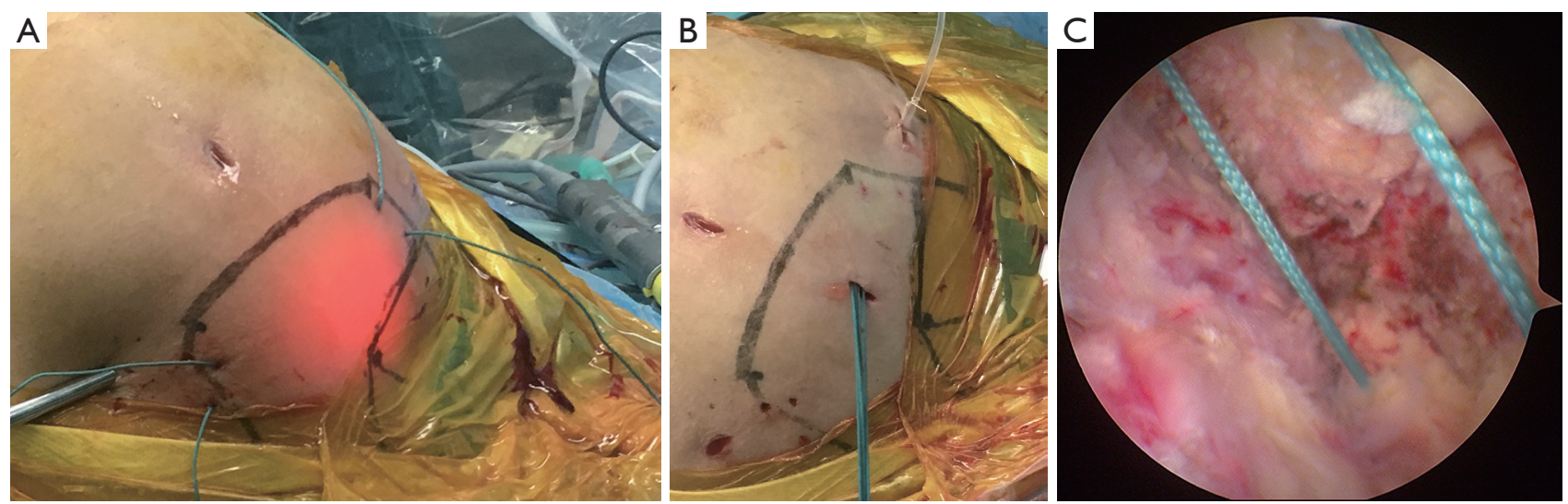

Figure 4 Suture management. (A) Outside look after two \#5 Ethibond sutures were pulled inside out; (B) a small incision of about 5 mm was made above the acromion for retrieving the sutures; $(\mathrm{C})$ the margin between the two fragments was gone. 
Table 2 The Constant score and VAS score

\begin{tabular}{lccc}
\hline Scale & Pre-operation & Post-operation & $P$ \\
\hline Constant score & $40.50 \pm 4.53$ & $75.60 \pm 5.17$ & $<0.001$ \\
VAS score & $5.20 \pm 1.14$ & $1.60 \pm 0.84$ & $<0.001$ \\
\hline
\end{tabular}

VAS, visual analog scale.

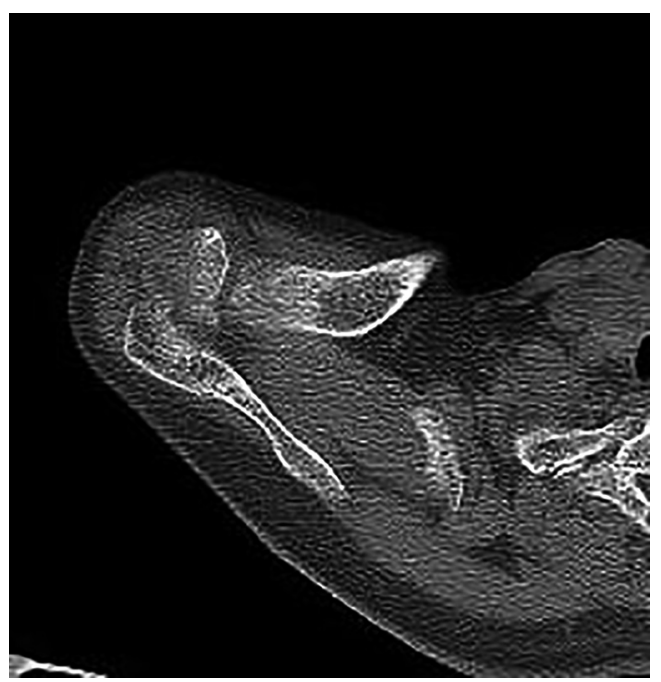

Figure $5 \mathrm{CT}$ scan 12 months after the surgery, showing bony union of the os acromiale.

superior part of the labrum without fixation.

\section{Statistical analysis}

All the data were analyzed in SPSS (version 19 Chicago, IL, USA). Paired $t$-test was used to compare the Constant scores and VAS scores. A P value of lower than 0.05 was considered statistically significant.

\section{Results}

All the patients were followed up with at our department. There were no severe complications, and all of them showed good recovery 6 months after the surgery. The average follow-up length was 28.7 months, ranging from 26 to 33 months. The average Constant score before surgery was $40.50 \pm 4.53$ points, and it was significantly improved to $75.60 \pm 5.17$ points after surgery. The average VAS score was reduced from $5.20 \pm 1.14$ points to $1.60 \pm 0.84$ (Table 2).

At the follow-up of 12 months, a CT scan was ordered.
All the patients showed the bony union of the os acromiale. On the CT scan, two small pits could be seen on the medial and lateral side of the acromion, which indicated the level of the os acromiale. The position of the os acromiale was good, and no evident sclerosis was found on the edges of the fragments (Figure 5).

\section{Discussion}

Anteroposterior (AP) and outlet radiographs were most commonly used in screening shoulder pain patients in our department. However, an axillary lateral radiograph was most useful in diagnosing os acromial $(6,10)$, which was not our routine examination. On MR images, os acromiale was frequently missed because it had a strong resemblance to a normal AC joint in the coronal or sagittal planes. It was most reliable to make the diagnosis of extremely proximal axial planes. For the first case of our series, those critical planes were overlooked before the surgery, and the diagnosis of os acromiale was not made until during the bursoscopy. Fortunately, it was well managed, and we gained experience and did not miss any os acromiale for the rest of the case series.

In the literature shown in Table 3, several surgical techniques were introduced. Warner et al. performed open excision of os acromiale on three patients, but 2 of them were unsatisfied with the outcomes and complained of persistent pain and deltoid weakness (3). However, Mudge et al. reported positive results in 4 out of six patients who received open fragment excision with rotator cuff wear (7). Pagnani et al. performed arthroscopic excision of os acromiale and demonstrated positive clinical functions for the operated shoulders (11). Wright et al. reported similar findings (9). However, there were no studies comparing the outcomes of open and arthroscopic excisions. Hutchinson and Veenstra performed arthroscopic subacromial decompression on three patients for impingement syndrome secondary to os acromiale (12). Despite this, 2 of them had recurrence of symptoms. Wright et al. performed a more aggressive subacromial decompression procedure on 13 shoulders with impingement symptoms due to os acromiale (9). The entire unstable anterior tip was almost resected, leaving only a thin superior shell. Twelve shoulders showed satisfactory clinical results. With inconsistent results of clinical outcomes, it remains controversial as to which of the above-mentioned options is superior. We believe that the small sample sizes of the previous studies might be a reason for the mixed results reported. 
Table 3 Surgical techniques reported in literature

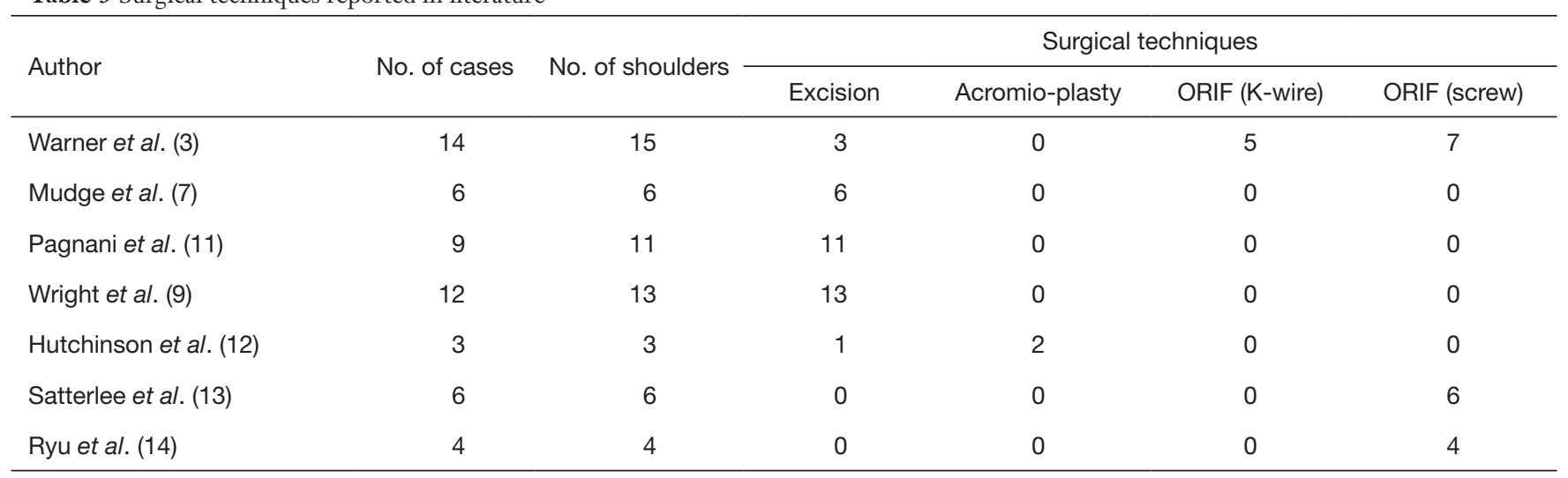

ORIF, open reduction and internal fixation.

ORIF surgery was another option for treating symptomatic os acromiale, and numerous techniques for ORIF have been reported including $\mathrm{K}$-wire fixation, screw fixation, and tension band fixation with cannulated screws (13-15). Complications of ORIF mainly include hardware irritation, acromial fracture, and nonunion.

In this study, we reported a novel surgical technique to fix os acromiale with polyester sutures arthroscopically. By using polyester sutures, some of the complications in previously reported studies can be avoided. First, polyester sutures are soft fixation materials, so hardware irritation did not exist in our case series, and there was no need to remove them. Second, the risk of acromial fracture was avoided. Cerclage with polyester sutures does not involve drilling holes in a small acromion, and os acromiale is not only technically challenging but also might cause a fracture. For the first case in our study, we intended to do a cerclage with wires, but we found that the bones easily got cut at the acromion due to osteoporosis. Polyester sutures were a better choice in this condition.

Tension strength and nonunion of the os acromiale were our greatest concern. Although we did not have direct evidence that polyester sutures could provide as strong stability as screws or wires, in a cadaver study, Shiu et al. reported that polyethylene sutures and stainless steel wires had similar biomechanical strength in the cannulated screw tension band fixation of os acromiale (16). In our clinical study, CT scans were carried out for each patient at the follow-up of 12 months, and we found that all the patients had a bony union of the os acromiale. These findings suggested that polyester sutures can provide enough tension for the fixation.
One limitation in this study was that we were not sure if fixation and bony union of the os acromiale helped in pain relief and function restoration of the shoulder. Burkhart reported that os acromiale may be completely unrelated to the true source of the patient's discomfort (17). In our case series, only three patients were diagnosed as isolated os acromiale. Although all 3 of them achieved satisfactory clinical outcomes, fixation was not the only procedure they underwent, and the effect of the routine subacromial debridement should not be overlooked. A well-designed randomized controlled trial is needed to achieve a more comprehensive understanding of this issue.

\section{Conclusions}

Polyester sutures could provide reliable strength in fixation of os acromiale without hardware irritation.

\section{Acknowledgments}

The authors thank Xi Chen and Jiaze Song for valuable suggestions.

\section{Footnote}

Conflicts of Interest: The authors have no conflicts of interest to declare.

Ethical Statement: The authors are accountable for all aspects of the work in ensuring that questions related to the accuracy or integrity of any part of the work are appropriately investigated and resolved. Ethical approval 
for this study was obtained from written informed consent signed by all the patients before the study. In the consent, all the patients were well informed and agreed that data regarding radiology examinations, pictures taken during the surgery, follow-up function scores, pathological examinations would be analyzed for any retrospective studies. The registration numbers are 0500703, 0639938, 0679015, 0745431, 0792030, 0851513, 0905400, 0909211, 0939212,0942022 .

\section{References}

1. Gruber W. Über die Arten der Adromialknochen und accidentellen Akromialgelenke. Arch Anat Physiolund Wissen Med 1863:373-93.

2. Atoun E, van Tongel A, Narvani A, et al. Arthroscopically assisted internal fixation of the symptomatic unstable os acromiale with absorbable screws. J Shoulder Elbow Surg 2012;21:1740-5.

3. Warner JJ, Beim GM, Higgins L. The treatment of symptomatic os acromiale. J Bone Joint Surg Am 1998;80:1320-6.

4. Nicholson GP, Goodman DA, Flatow EL, et al. The acromion: morphologic condition and age-related changes. A study of 420 scapulas. J Shoulder Elbow Surg 1996;5:1-11.

5. Sammarco VJ. Os acromiale: frequency, anatomy, and clinical implications. J Bone Joint Surg Am 2000;82:394-400.

6. Kurtz CA, Humble BJ, Rodosky MW, et al. Symptomatic os acromiale. J Am Acad Orthop Surg 2006;14:12-9.

7. Mudge MK, Wood VE, Frykman GK. Rotator cuff

Cite this article as: Guo DM, Li ZX, Wang Q, Song HH. Fixation of os acromiale using polyester sutures: a novel surgical treatment. Ann Transl Med 2019;7(18):448. doi: 10.21037/ atm.2019.08.58 tears associated with os acromiale. J Bone Joint Surg Am 1984;66:427-9.

8. Johnston PS, Paxton ES, Gordon V, et al. Os acromiale: a review and an introduction of a new surgical technique for management. Orthop Clin North Am 2013;44:635-44.

9. Wright RW, Heller MA, Quick DC, et al. Arthroscopic decompression for impingement syndrome secondary to an unstable os acromiale. Arthroscopy 2000;16:595-9.

10. Peckett WR, Gunther SB, Harper GD, et al. Internal fixation of symptomatic os acromiale: a series of twenty-six cases. J Shoulder Elbow Surg 2004;13:381-5.

11. Pagnani MJ, Mathis CE, Solman CG. Painful os acromiale (or unfused acromial apophysis) in athletes. J Shoulder Elbow Surg 2006;15:432-5.

12. Hutchinson MR, Veenstra MA. Arthroscopic decompression of shoulder impingement secondary to Os acromiale. Arthroscopy 1993;9:28-32.

13. Satterlee CC. Successful osteosynthesis of an unstable mesoacromion in 6 shoulders: a new technique. J Shoulder Elbow Surg 1999;8:125-9.

14. Ryu RK, Fan RS, Dunbar WH 5th. The treatment of symptomatic os acromiale. Orthopedics 1999;22:325-8.

15. Harris JD, Griesser MJ, Jones GL. Systematic review of the surgical treatment for symptomatic os acromiale. Int J Shoulder Surg 2011;5:9-16.

16. Shiu B, Song $\mathrm{X}$, Iacangelo A, et al. Os acromiale fixation: a biomechanical comparison of polyethylene suture versus stainless steel wire tension band. J Shoulder Elbow Surg 2016;25:2034-9.

17. Burkhart SS. Os acromiale in a professional tennis player. Am J Sports Med 1992;20:483-4. 